DRAFT VERSION OCTOBER 23, 2018

Preprint typeset using LTEX style emulateapj v. 11/10/09

\title{
X-RAY EMISSION FROM THE DOUBLE-BINARY OB-STAR SYSTEM QZ CAR (HD 93206)
}

\author{
E. R. Parkin ${ }^{1,2}$, P. S. Broos ${ }^{3}$, L. K. Townsley ${ }^{3}$, J. M. Pittard ${ }^{2}$, A. F. J. Moffat ${ }^{4}$, Y. Nazé ${ }^{1}$, G. RAuw ${ }^{1}$ L. M. Oskinova $^{5}$, \\ W. L. WALDRON ${ }^{6}$ \\ ${ }^{1}$ Institut d'Astrophysique et de Géophysique, Université de Liège, 17, Allée du 6 Août, B5c, B-4000 Sart Tilman, Belgium \\ ${ }^{2}$ School of Physics and Astronomy, The University of Leeds, Woodhouse Lane, Leeds LS2 9JT, UK \\ ${ }^{3}$ Department of Astronomy and Astrophysics, Pennsylvania State University, 525 Davey Laboratory, University Park, PA 16802 \\ ${ }^{4}$ Département de Physique, Université de Montreal, C.P. 6128, Succ. Centre-Ville, Montreal, QC H3C 3J7, Canada \\ ${ }^{5}$ Institute for Physics and Astronomy, University of Potsdam, 14476 Potsdam, Germany and \\ ${ }^{6}$ Eureka Scientific Inc., 2452 Delmer Street, Oakland, CA 94602, USA \\ Draft version October 23, 2018
}

\begin{abstract}
X-ray observations of the double-binary OB-star system QZ Car (HD 93206) obtained with the Chandra $\mathrm{X}$-ray Observatory over a period of roughly 2 years are presented. The orbit of systems $\mathrm{A}\left(\mathrm{O} 9.7 \mathrm{I}+\mathrm{b} 2 \mathrm{v}, \mathrm{P}_{\mathrm{A}}=\right.$ $21 \mathrm{~d})$ and $\mathrm{B}\left(\mathrm{O} 8 \mathrm{III}+\mathrm{o} 9 \mathrm{v}, \mathrm{P}_{\mathrm{B}}=6 \mathrm{~d}\right)$ are reasonably well sampled by the observations, allowing the origin of the $\mathrm{X}$-ray emission to be examined in detail. The X-ray spectra can be well fitted by an attenuated three temperature thermal plasma model, characterised by cool, moderate, and hot plasma components at $k T \simeq 0.2,0.7$, and 2 $\mathrm{keV}$, respectively, and a circumstellar absorption of $\simeq 0.2 \times 10^{22} \mathrm{~cm}^{-2}$. Although the hot plasma component could be indicating the presence of wind-wind collision shocks in the system, the model fluxes calculated from spectral fits, with an average value of $\simeq 7 \times 10^{-13} \mathrm{erg} \mathrm{s}^{-1} \mathrm{~cm}^{-2}$, do not show a clear correlation with the orbits of the two constituent binaries. A semi-analytical model of QZ Car reveals that a stable momentum balance may not be established in either system A or B. Yet, despite this, system B is expected to produce an observed X-ray flux well in excess of the observations. If one considers the wind of the O8 III star to be disrupted by mass transfer the model and observations are in far better agreement, which lends support to the previous suggestion of mass-transfer in the $08 \mathrm{III}+09 \mathrm{v}$ binary. We conclude that the X-ray emission from QZ Car can be reasonably well accounted for by a combination of contributions mainly from the single stars and the mutual wind-wind collision between systems A and B.
\end{abstract}

Subject headings: hydrodynamics - stars:early-type - X-rays:stars - stars:binaries - stars:winds, outflows stars:individual(QZ Carinae)

\section{INTRODUCTION}

Residing within the Great Carina Nebula at a distance of 2.3 kpc (Allen \& Hillier 1993; Walborn 1995; Smith 2002, see also Smith 2006), the multiple star system QZ Car (HD 93206) is the brightest object in the Collinder 228 star cluster, in the older southern part of the Nebula. Via independent observations, the presence of two systems of periodically variable lines in the spectrum led Leung et al. (1979) and Morrison \& Conti (1979) to conclude that there were four stars present, where the period of the stronger line variability was due to the $\sim 20$ day binary (hereafter system A) and the period of the weaker lines corresponding to the $\sim 6$ day period eclipsing binary (hereafter system B). Despite this great success two of the components of the system remain undetected. The mass functions derived for the separate binary systems suggest that the eclipsing binary component with undetected lines is more massive than its binary companion, whereas in the longer period, non-eclipsing binary the unseen companion is a few times smaller than the primary component and therefore most likely has unobservable lines. A schematic of QZ Car is shown in Fig. 1 and system and stellar parameters are noted in Tables 1 and 2, respectively. Based on the Roche-lobe filling factor 1 , Leung et al. (1979) suggested that the stars in system B have undergone some mass exchange. Morrison \& Conti (1980) also noted that there is evidence of

\footnotetext{
email: parkin@mso.anu.edu.au

1 The parameters in Table 2 give Roche-lobe filling factors for stars B1
} and $\mathrm{B} 2$ of $\simeq 1.0$ and 0.4 , respectively. substantial mass-loss from the primary star in system B due to a systematic difference in velocity between He I and Si IV, which from an evolutionary point of view makes it the most interesting star in this system.

Little is known about the mutual orbit of systems A and B. The results of Leung et al. (1979) and Morrison \& Conti (1980) were in agreement that the orbital period of the superbinary must be $\lesssim 25$ yrs. Yet this was based on the assumption that at the time of their observations the system was at quadrature, or apastron in an eccentric orbit. The speckle observations of Mason et al. (1998) were unsuccessful in spatially resolving the components of HD 93206, as were the more sensitive FGS1r observations of Nelan et al. (2004). Therefore, the non-resolution of the system places an upper limit of $\simeq 35$ au on the projected separation of the two binary systems.

In this paper we report on the recent detection of X-ray emission from QZ Car. For single massive stars it is widely accepted that (soft) X-ray emission is generated by embedded wind shocks (EWSs) which are produced by the inherent instability of the line-driving mechanism (e.g. Owocki et al. 1988). Early X-ray observations of massive stars in binary systems revealed them to be over-luminous compared to the expected cumulative luminosity of the separate stars (Pollock 1987; Chlebowski \& Garmany 1991). The addi-

\footnotetext{
${ }^{2}$ More recent results (Oskinova 2005; Nazé 2009; Naze et al. 2011) suggest that only prominent colliding winds binary systems are significantly over-luminous in X-rays.
} 


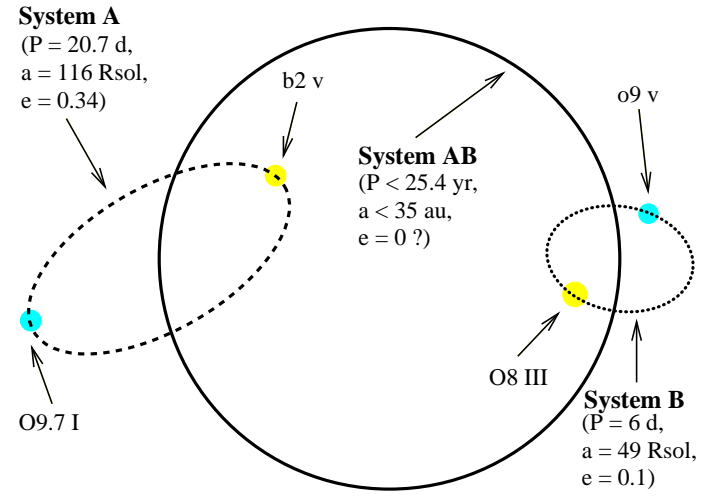

FIG. 1.- Schematic diagram of the multiple star system QZ Car. For further details see Tables 1 and 2 For system AB the projected semi-major axis is quoted. Note that this schematic is not to scale.

tional luminosity in this case is the result of wind-wind collision shocks (e.g. Stevens et al. 1992; Pittard \& Stevens 1997; Parkin \& Pittard 2008). For QZ Car, further additional X-ray emission may be contributed by the mutual wind-wind collision between the two binary systems (MWC). The observed flux may therefore be a complex cocktail of X-ray emission from different sources, and disentangling its origin(s) is not straightforward. We note that the central multiple star system is surrounded by a subcluster of faint X-ray emitting pre-main sequence stars, for which we refer the reader to Townsley et al. (2011) for a detailed analysis.

The X-ray observations of QZ Car were obtained with the Chandra X-ray observatory (hereafter Chandra) as part of the Chandra Carina Complex Project (CCCP)(Townsley et al. 2011; Broos et al. 2011). The X-ray spectra can be reasonably well fitted by three-temperature plasma models with a hot component at $\simeq 2 \mathrm{keV}$. This, combined with the fact that the observed fluxes appear to be over-luminous in comparison to the total X-ray emission expected from the single stars, may be indicating the presence of shock heated plasma from windwind collisions. However, attempts to match the best-fit parameters from the spectral fits to the periods of either system A or B do not reveal any strong correlation. To aid in the interpretation of the observations a semi-analytical model is constructed which indicates that although normal wind-wind collision shocks are not expected, unless the wind of the O8 III star is suppressed the model overpredicts the observed X-ray emission by a factor of $\sim 10-20$. The suppressant in this case could be mass transfer from the O8 III star (Leung et al. 1979). We conclude that the dominant contributions to the observed X-ray flux are the single stars and the MWC. The remainder of this paper is structured as follows: in $\S 2$ we present the observations, $\S 3$ describes the results from spectral fitting, $\S 4$ details a semi-analytical model of QZ Car. The results from this work are discussed in $\S 5$ and we close with a summary of our conclusions in $\S 6$.

\section{OBSERVATIONS}

A total of nine observations over a period of roughly two years, and combining relatively on-axis and far off-axis exposures taken with both the I-array and the S-array, have been obtained for QZ Car (Table 3). Due to the brightness of the central source the I-array observation (Obs ID 9482) was affected by photon pile-up, which we account for in our analysis (see $\S$ 3). The I-array observation alone would have provided a single snapshot of QZ Car; however, during the CCCP
TABLE 1

SYSTEM PARAMETERS FOR QZ CAR.

\begin{tabular}{lllllll}
\hline System & Components & $P$ & $\begin{array}{l}a \\
(\mathrm{R} \odot)\end{array}$ & $e$ & $\begin{array}{l}\omega \\
\left({ }^{\circ}\right)\end{array}$ & $\begin{array}{l}i \\
\left({ }^{\circ}\right)\end{array}$ \\
\hline $\mathrm{A}$ & $\mathrm{A} 1+\mathrm{A} 2$ & $20.72 \mathrm{~d}$ & 116 & 0.34 & 141 & 60 \\
$\mathrm{~B}$ & $\mathrm{~B} 1+\mathrm{B} 2$ & $5.999 \mathrm{~d}$ & 49 & 0.1 & $\simeq 20$ & 86 \\
$\mathrm{AB}$ & $\mathrm{A}+\mathrm{B}$ & $\lesssim 25.4 \mathrm{yrs}$ & $\lesssim 8687$ & 0.0 & 0 & 60 \\
\hline
\end{tabular}

NOTE. $-P$ is the period of the orbit, $a$ is the semi-major axis of the orbit, $e$ is the orbital eccentricity, $\omega$ is the longitude of periastron, and $i$ is the inclination angle of the orbital plane (measured against the pole). Orbital periods are taken from Mayer et al. (2001), e's and $\omega$ 's from Morrison \& Conti (1980), and $i$ 's from Leung et al. (1979). For system AB the projected semi-major axis is quoted. We note that $e=0.0$ is only a preliminary assumption for system $\mathrm{AB}$, and such long-period systems can in fact have $0.000 \lesssim e \lesssim 0.999$

QZ Car has been observed by the S-array CCDs on eight separate occasions, and in some cases with a considerable exposure time (e.g. $88 \mathrm{ks}$ for Obs ID 6402). Fortunately for the current investigation this considerably expands the available dataset.

Source and spectrum extraction were performed using ACIS EXTRACT Broos et al. 2002, 2007, 2010; Townsley et al. 2006), an IDL-based package developed for processing ACIS data. For each observation, a background spectrum was taken from an annulus around QZ Car, and the resulting background subtracted source spectrum was binned to achieve a signal-to-noise ratio of 3 . Note that the far offaxis (ACIS-S) extractions of QZ Car encompass a nearby subcluster of low-mass stars. However, these potential contaminants to the off-axis QZ Car spectra are very weak when resolved in the on-axis observation, and the off-axis extractions show no indication of a large flare from one of these companions: i) the median energy shows relatively minor changes, and, ii) Kolmogorov-Smirnov (KS) tests on the individual lightcurves do not reveal any considerable evidence for variability.

\section{RESULTS}

The $0.5-8 \mathrm{keV}$ spectra were fitted using v12.5.1 of XSPEC (Arnaud 1996). To model the emission we use the apec thermal plasma model for collisionally-ionized gas (Smith et al. 2001), and to account for attenuation we adopted the tbabs photoelectric absorption model (see Wilms et al.2000). In all calculations the abundances were kept fixed at the solar values (Anders \& Grevesse 1989). To fit the spectra we use a three temperature combination, tbabs ISM $\times$ tbabs $($ apec + apec + apec), with an ISM absorption component, tbabs $s_{\mathrm{ISM}}$, fixed at $0.35 \times 10^{22} \mathrm{~cm}^{-2}$ (Povich et al. 2011). By separating the column into ISM and circumstellar components we gain more information about variations in the local absorption, which is a particularly useful approach when studying the windwind collision in binary systems (e.g. De Becker et al. 2005; Pittard \& Parkin 2010). Similarly, the separate emission components can be used to search for correlations with the components of the system (i.e. EWSs, wind-wind collision regions (WCRs), and the MWC). The physical interpretation of the adopted model combination assumes that the circumstellar absorption to all emission components is the same. As

\footnotetext{
3 http://heasarc.gsfc.nasa.gov/docs/xanadu/xspec/

4 A more physically meaningful model combination would incorporate separate absorption components for each emission component (i.e. spatially distinct emission regions). However, due to limited statistics attempts to fit the spectra with such a model combination were unsuccessful.
} 

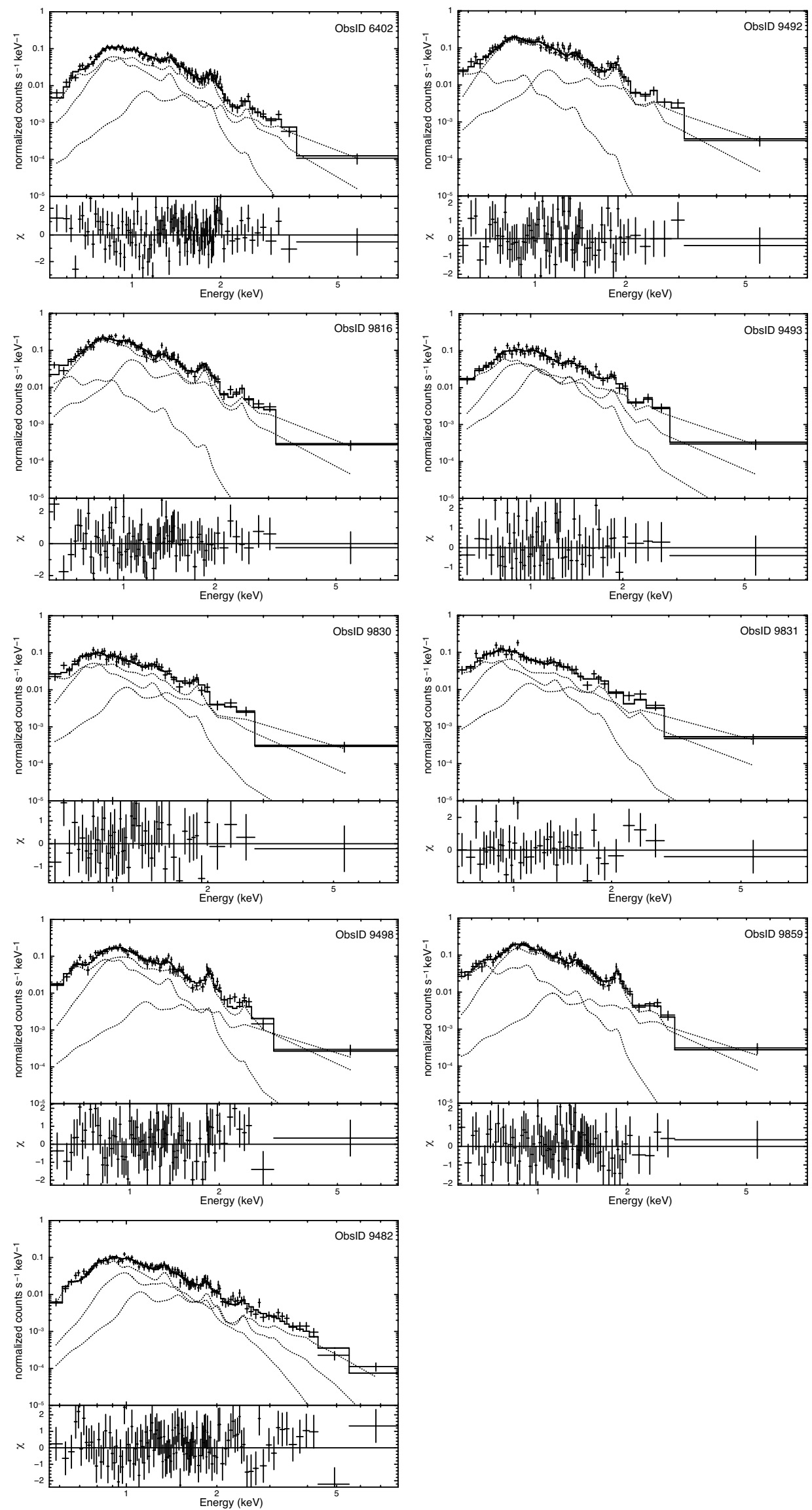

FIG. 2. - 0.5-8 keV X-ray spectra of QZ Car with best fit models attained with the combination $t b a b s_{\text {ISM }} \times t b a b s(a p e c+a p e c+a p e c)$. 
noted in $\S 2$. Obs ID 9482 was affected by photon pile-up 5 . which we correct for using an additional pileup model (Davis 2001). The pileup parameter fr_time was frozen to $3.31 \mathrm{~s}$ to account for exposure time discarded during the standard data cleaning process 6 ; the best-fit values for the thawed $\alpha$ and psffrac parameters were found to be 0.954 and 0.633 .

A visual inspection of the spectra shows that a three temperature thermal plasma model provides a reasonably good fit to the data (Fig. 2). In general there is a cool component at $k T_{1} \simeq 0.2 \mathrm{keV}$, a moderate temperature component at $k T_{2} \simeq 0.7 \mathrm{keV}$, a hot component at $k T_{3} \simeq 2 \mathrm{keV}$, a column density, $N_{\mathrm{H}} \simeq 0.2 \times 10^{22} \mathrm{~cm}^{-2}$, and a $0.5-8 \mathrm{keV}$ flux $\simeq 7 \times 10^{-13} \mathrm{erg} \mathrm{s}^{-1} \mathrm{~cm}^{-2}$. Comparing these results to those for QZ Car in Naze et al. (2011) we see that there is agreement in the circumstellar column and in the presence of a moderate temperature component at $\simeq 0.7 \mathrm{keV}$. However, the derived flux and temperature of the hotter plasma component are notably higher in this work. These differences are likely due to the use of three-temperature fits in this work, whereas Naze et al. apply a two-temperature fit?. We note that two-temperature fits were also examined, however, statistically better results could be attained for all observations using three-temperature spectral fits. For example, for ObsID 6402, the reduced chi-squared attained from twotemperature and three-temperature fits were 1.84 and 1.33 , respectively. In both cases the highest temperature component was at $k T \simeq 2.1 \mathrm{keV}$. The higher chi-squared in the case of the two-temperature model was due to poorer fit to the spectrum at energies $\lesssim 1 \mathrm{keV}$. Adding the third temperature component significantly remedied this.

From the best-fit parameters in Table 4 (see also Fig. 3) one sees that $k T_{1}$ and $k T_{2}$ appear to be reasonably well constrained. However, in constrast $k T_{3}$ and $N_{\mathrm{H}}$ do not. Recalling that $N_{\mathrm{H}}$ is intended to account for the circumstellar absorption to potentially numerous regions of X-ray emitting plasma, this is unsurprising. Despite this, using the conversion factor of $N_{\mathrm{H}}=A_{\mathrm{v}} \times\left(1.9 \times 10^{21}\right) \mathrm{cm}^{-2}($ e.g. $\operatorname{Cox} 2000)$, there is good agreement with the visual extinction from the observed colour indices $\left(A_{\mathrm{v}}=1.2 \mathrm{mag}\right.$; Herbst 1976; Leung et al. 1979). Comparisons of the X-ray spectra of QZ Car against two-temperature fits to single and binary stars of similar spectral type in Carina (Naze et al. 2011) are inconclusive in so much as they do not directly support/rule-out the presence of wind-wind collision shocks based solely on the spectral shape and the derived plasma temperatures (i.e. the presence of a hot component with $k T \gtrsim 1 \mathrm{keV}$ ).

Examining the variation of the best-fit parameters plotted against the orbital phases of the binary systems we see that both $k T_{1}$ and $k T_{2}$ remain relatively constant across all observations (Fig. 3). A tentative correlation between $k T_{3}$ and system A could be suggested although the errors are quite large (due to limited statistics in the spectra at higher energies) and one could equally favour a null result. Additionally, an in-

5 http://cxc.harvard.edu/ciao/why/pileup_intro.html 6 The Chandra ABC Guide to Pileup (http://cxc.harvard.edu/ciao/download/doc/pileup_abc.pdf describes the correction to fr_time that is required for all ACIS sources.

${ }^{7}$ The quality of the data constrains the complexity of the model that can be applied in spectral fitting. Naze et al. (2011) adopted two-temperature model fits to perform a consistent analysis of the entire $O B$ star sample from the CCCP, which consists of data of varying quality. In the present paper, we aim to perform a more detailed analysis of QZ Car, for which the data is of sufficient quality to permit meaningful results from the use of a threetemperature model. crease in $N_{\mathrm{H}}$ would be expected as higher temperature plasma close to the apex of the WCR(s) comes into view, which is not seen in the fits. Finally, we note that the $0.5-8 \mathrm{keV}$ fluxes calculated from the spectral fits do not show any clear correlation with the orbit of either system A or B.

\section{A MODEL OF QZ CAR}

The results of the analysis so far do not highlight any obvious link between the observed X-ray emission and the orbit of either binary. However, with potentially multiple sources of X-rays it is possible that any signature of the orbit may be smeared-out in the cumulative emission. To better constrain the rôles of the various components of the system, and their contribution to the total emission, we now construct a semianalytical model of QZ Car.

\subsection{Intrinsic $X$-ray emission}

Firstly, we estimate the contribution to the X-ray luminosity from embedded wind shocks (EWSs) using the canonical relation $L_{\mathrm{X}}=10^{-7} L_{\mathrm{bol}}$, which amounts to a flux of $\simeq$ $4.4 \times 10^{-13} \mathrm{erg} \mathrm{s}^{-1} \mathrm{~cm}^{-2}$. Then, following Pittard \& Stevens (2002), we can estimate the X-ray flux from the wind-wind collisions using the simple relation:

$$
f_{\mathrm{Xi}}=\frac{1}{8 \pi D^{2}} \dot{M}_{\mathrm{i}} v_{\mathrm{i}}^{2} \frac{\Xi_{\mathrm{i}}}{\chi_{\mathrm{i}}}
$$

where $D$ is the distance to $\mathrm{QZ}$ Car (taken to be $2.3 \mathrm{kpc}$ ), $\Xi$ is the fractional wind kinetic power normal to the contact discontinuity, $\chi$ is the cooling parameter $\left(=v_{8}^{4} d_{12} / \dot{M}_{-7}\right.$, see Stevens et al. 1992), and the subscript $i$ denotes the contributing component. The parameter $\Xi$ is dependent on the wind momentum ratio of the system, $\eta_{\mathrm{ij}}=\left(\dot{M}_{\mathrm{j}} v_{\mathrm{j}}\right) /\left(\dot{M}_{\mathrm{i}} v_{\mathrm{i}}\right)$, where the subscript $j$ denotes the component index of the binary companion. For $\eta_{\mathrm{ij}}=(0.01,0.1,1.0), \Xi_{\mathrm{i}}=$ $(0.0042,0.033,0.167)$ and $\Xi_{j}=(0.564,0.403,0.167)$, i.e. the value of $\Xi$ is higher for the weaker wind because a greater fraction of that wind collides close to the shock normal. The parameter $\chi$ is the ratio of the characteristic flow time to the cooling time; if $\chi \lesssim 1$ the post-shock gas is radiative, whereas if $\chi \gg 1$ the post-shock gas is adiabatic. Note that in the case where $\chi<1$ we set $\chi=1$ to satisfy energy conservation.

In systems $A$ and $B$ the separation of the stars is relatively small, and the stellar winds may not have reached their terminal velocities. This has consequences for the position of the momentum balance surface between the stars (if one exists). If we assume that the wind velocity follows a $\beta$-velocity law (i.e. $\left.v(r)=v_{\infty}\left(1-R_{*} / r\right)^{\beta}\right)$, and set $\beta=1$ for simplicity, we can calculate the position of the momentum balance point by numerical solution of the following equation for the distance from star $\mathrm{i}$ to the momentum balance point (along the line-of-centres), $r$,

$$
\frac{\dot{M}_{\mathrm{i}} v_{\infty \mathrm{i}}}{r^{2}}\left(1-\frac{R_{* \mathrm{i}}}{r}\right)=\frac{\dot{M}_{\mathrm{j}} v_{\infty \mathrm{j}}}{\left(d_{\mathrm{sep}}-r\right)^{2}}\left(1-\frac{R_{* \mathrm{j}}}{d_{\mathrm{sep}}-r}\right) .
$$

It is then straight-forward to calculate the effective values of $\eta, \Xi, \chi$, and $f_{\mathrm{Xi}}$ from the shocked gas of each wind, where the preshock wind speed along the line-of-centres rather than the terminal wind speed is used in the calculations. The distance from the star to the shock rather than the binary separation is used to calculate $\chi$.

We can repeat this process for the MWC. For this purpose we approximate the mass-loss rates and terminal wind speeds 
TABLE 2

STELlar PARAMETERS FOR QZ CAR.

\begin{tabular}{llllllll}
\hline Component & Sp. Type & $\begin{array}{l}T_{\text {eff }} \\
(\mathrm{K})\end{array}$ & $\begin{array}{l}R_{*} \\
\left(\mathrm{R}_{\odot}\right)\end{array}$ & $\begin{array}{l}M_{*} \\
\left(\mathrm{M}_{\odot}\right)\end{array}$ & $\begin{array}{l}L_{*} \\
\left(\log \left[L_{*} / \mathrm{L}_{\odot}\right]\right)\end{array}$ & $\begin{array}{l}\dot{M} \\
\left(\mathrm{M}_{\odot} \mathrm{yr}^{-1}\right)\end{array}$ & $\begin{array}{l}v_{\infty} \\
\left(\mathrm{km} \mathrm{s}^{-1}\right)\end{array}$ \\
\hline A1 & O9.7 I & 32000 & 22.5 & 40 & 5.7 & $2.2 \times 10^{-6}$ & 2140 \\
A2 & b2 v & 20000 & 6.0 & 10 & 3.7 & $2.4 \times 10^{-9}$ & 1040 \\
B1 & 08 III & 32573 & 26.9 & 14.1 & 5.3 & $5.2 \times 10^{-7}$ & 2220 \\
B2 & 09 v & 32463 & 8.9 & 28 & 4.9 & $6.4 \times 10^{-8}$ & 2850 \\
\hline
\end{tabular}

NotE. - For consistency we adopt the labelling of Mayer et al. (2001) for the system components. The spectral types for components A1 and B1 were determined from observations by the OWN Team (R. Barba, private communication). The values of $T_{\text {eff }}, R_{*}$, and $M_{*}$ for components A1 and B2 are taken from Leung et al. (1979), for component B1 values were taken from Martins et al. (2005), and for component A2 the respective values have been estimated by a comparison to objects of similar spectral type in Prinia (1989). The values of $v_{\infty}$ are calculated as $2.6 v_{\text {esc }}$ for components A1, B1, and B2, and as $1.3 v_{\text {esc }}$ for component A2 (based on $T_{\text {eff }}$ ), where $v_{\text {esc }}=\sqrt{2 G M_{*} / R_{*}}$. The $\dot{M}$ values are calculated using the cooking recipe from Vink et al. (2000). We note that the spectral types of components A2 and B2 are not based on true spectral classifications (i.e. direct detection in the optical spectrum), and are in fact based on photometric and/or colour information. Therefore, we use lowercase letters to denote the spectral type of components A2 and B2.

TABLE 3

SUMMARY OF THE OBSERVATIONS.

\begin{tabular}{llllllll}
\hline Obs ID & CCD & $\begin{array}{l}\theta \\
\left({ }^{\prime}\right)\end{array}$ & Date & $\begin{array}{l}T \\
(\mathrm{ks})\end{array}$ & $\begin{array}{l}R \\
\left(\mathrm{ks}^{-1}\right)\end{array}$ & $\phi_{\mathrm{A}}$ & $\phi_{\mathrm{B}}$ \\
\hline 6402 & $\mathrm{~S} 2$ & 16.01 & 30 Aug 2006 & 87 & 76.5 & 0.10 & 0.07 \\
9492 & $\mathrm{~S} 3$ & 14.04 & 12 Feb 2008 & 20 & 119.0 & 0.68 & 0.50 \\
9816 & $\mathrm{~S} 3$ & 14.04 & 15 Feb 2008 & 21 & 138.5 & 0.81 & 0.94 \\
9493 & $\mathrm{~S} 2$ & 17.23 & 25 Feb 2008 & 20 & 83.1 & 0.32 & 0.70 \\
9830 & S2 & 17.23 & 28 Feb 2008 & 20 & 73.3 & 0.45 & 0.14 \\
9831 & S2 & 17.23 & 1 Mar 2008 & 16 & 78.4 & 0.52 & 0.41 \\
9498 & S3 & 18.94 & 24 May 2008 & 32 & 118.3 & 0.61 & 0.53 \\
9859 & S3 & 18.94 & 31 May 2008 & 28 & 129.4 & 0.91 & 0.57 \\
9482 & I3 & 3.41 & 18 Aug 2008 & 57 & 73.3 & 0.75 & 0.83 \\
\hline
\end{tabular}

NOTE. $-\phi_{\mathrm{A}}$ and $\phi_{\mathrm{B}}$ are the corresponding orbital phases for system A and system B, respectively, calculated using the ephimerides of Maver et al. (2001). The time of periastron used for system A is taken as JD 2442530.49, which refines the time of periastron determined by Morrison \& Conti (1980) using the more recent observations of Mayer et al. (2001). For system B, the ephemeris is extrapolated from the minimum observed by Mayer et al. (1998) at JD 2448687.16. $\theta$ is the off-axis angle, $T$ is the exposure time, and $R$ is the count rate for each observation. The CCCP ACIS source label and official source name for QZ Car are C2_1111 and 104422.91-595935.9, respectively.

for systems A and B as,

$$
\begin{aligned}
\dot{M}_{\mathrm{A}} & =\dot{M}_{\mathrm{A} 1}+\dot{M}_{\mathrm{A} 2}, \\
\dot{M}_{\mathrm{B}} & =\dot{M}_{\mathrm{B} 1}+\dot{M}_{\mathrm{B} 2}, \\
v_{\mathrm{A}} & =\left(\frac{\dot{M}_{\mathrm{A} 1} v_{\mathrm{A} 1}^{2}+\dot{M}_{\mathrm{A} 2} v_{\mathrm{A} 2}^{2}}{\dot{M}_{\mathrm{A} 1}+\dot{M}_{\mathrm{A} 2}}\right)^{1 / 2}, \\
v_{\mathrm{B}} & =\left(\frac{\dot{M}_{\mathrm{B} 1} v_{\mathrm{B} 1}^{2}+\dot{M}_{\mathrm{B} 2} v_{\mathrm{B} 2}^{2}}{\dot{M}_{\mathrm{B} 1}+\dot{M}_{\mathrm{B} 2}}\right)^{1 / 2},
\end{aligned}
$$

Parameter values pertaining to the X-ray emission calculations for the MWC are listed in Table 5. We note that Eqs 3 , 6 should provide a reasonable approximation as, due to the relatively large separation of system $\mathrm{AB}$, the stellar winds should have had sufficient time to mix.

At all orbital phases in systems A and B a wind-wind momentum balance does not occur and the wind of the weaker star is completely crushed by the stronger opponen 8 . Therefore, the values of $\Xi_{\mathrm{A} 2}$ and $\Xi_{\mathrm{B} 2}$ are set to zero (i.e. no contribution to the X-ray emission) and $\Xi_{\mathrm{A} 1}$ and $\Xi_{\mathrm{B} 1}$ are instead approximated as the fractional solid angle subtended by the

\footnotetext{
${ }^{8}$ The weaker star may radiatively brake the incoming wind, permitting a ram pressure balance (Gayley et al. 1997). This is unlikely to be effective for system A (due to the relatively low luminosity of component A2 compared to component A1), but may be effective in system B.
}

face of their respective companion star,

$$
\Xi_{i}=\frac{1}{4 \pi}\left(1-\cos \left\{\tan ^{-1}\left(\frac{R_{* \mathrm{j}}}{d_{\text {sepi }-\mathrm{j}}}\right)\right\}\right) .
$$

Fig. 4 shows the variation of the pre-shock velocities, $\chi$ 's, $\Xi$ 's, and $k T$ with orbital phase. Note that, to avoid confusion with references to the intrinsic emission from the individual stars, the characteristics of component A1's wind colliding against its opposing star (component A2) is referred to as component A1-O. The same nomenclature is adopted for component B1. The characteristic energy of the emitted Xrays is given by $k T \simeq 1.17 v_{8}^{2} \mathrm{keV}$, where $v_{8}$ is the pre-shock velocity in units of $10^{8} \mathrm{~cm} \mathrm{~s}^{-1}$. For now, the preshock velocities of component B1-O are calculated assuming that it drives a wind towards its binary companion, rather than the system being semi-detached. Later $(\S 4.3$ we consider the possibility of zero colliding winds emission from system $B$.

Evidently, terminal wind speeds are not reached prior to collision (see Table 2). The cooling parameters, $\chi$ 's, for components A1-O and B1-O are sufficiently high for the postshock gas to be adiabatic at phases close to apastron, whereas they may become radiative $(\chi \lesssim 1)$ around periastron. The value of $\Xi$ for component B1-O is the highest at all orbital phases, representative of the larger fractional solid angle subtended by its companion star in comparison to component A1-O (see Eq77). The separation of systems A and B is sufficiently large that for the MWC the stellar winds will have reached their terminal velocities when they collide and this factor, combined with the low post-shock densities, leads to adiabatic shocks $\left(\chi_{\mathrm{A}}=570\right.$ and $\left.\chi_{\mathrm{B}}=2860\right)$.

With a range of pre-shock velocities, the X-ray spectrum for QZ Car may well be dominated by emission from different shocked plasma components at different energies. Approximating the mean $k T$ to be roughly half of the maximum value (to account for shock obliquity downstream from the apex of the WCR) we see that the predicted values for component A1$\mathrm{O}$ (Fig. 4 d) are slightly lower than those derived for the hot plasma component from the spectral fits (Fig. 3 and Table 4) which has a mean temperature of $2.00 \mathrm{keV}$. In contrast, the mean temperatures from the $\mathrm{MWC}$, where $k T$ 's are $\simeq 2.7$ and $3.1 \mathrm{keV}$ for system A and B respectively, are higher than the observationally determined value. We note that all the values are higher than our adopted mean plasma temperature for EWSs of $k T=0.25 \mathrm{keV}$ (e.g. Owocki \& Cohen 1999).

The intrinsic X-ray flux from the individual shocked winds is shown in Fig. 44. Component B1-O has the highest intrinsic $\mathrm{X}$-ray luminosity, and so system $\mathrm{B}$ will dominate the $\mathrm{X}$-ray emission if one assumes that the WCR has not been disrupted 
TABLE 4

RESULTS FROM SPECTRAL FITTING.

\begin{tabular}{|c|c|c|c|c|c|c|c|c|c|}
\hline Obs ID & $\begin{array}{l}N_{\mathrm{H} 1} \\
\left(10^{22} \mathrm{~cm}^{-2}\right)\end{array}$ & $\begin{array}{l}k T_{1} \\
(\mathrm{keV}) \\
\end{array}$ & $\begin{array}{l}\text { Norm }_{1} \\
\left(10^{-4} \mathrm{~cm}^{-5}\right)\end{array}$ & $\begin{array}{l}k T_{2} \\
(\mathrm{keV}) \\
\end{array}$ & $\begin{array}{l}\operatorname{Norm}_{2} \\
\left(10^{-4} \mathrm{~cm}^{-5}\right)\end{array}$ & $\begin{array}{l}k T_{3} \\
(\mathrm{keV}) \\
\end{array}$ & $\begin{array}{l}\operatorname{Norm}_{3} \\
\left(10^{-4} \mathrm{~cm}^{-5}\right)\end{array}$ & $\begin{array}{l}0.5-8 \mathrm{keV} \text { flux } \\
\left(10^{-13} \mathrm{erg} \mathrm{cm}^{-2} \mathrm{~s}^{-1}\right)\end{array}$ & $\begin{array}{l}\chi^{2} \\
\text { (d.o.f) }\end{array}$ \\
\hline 6402 & $0.25_{0.05}^{0.05}$ & $0.26_{0.06}^{0.05}$ & $23.4_{8.4}^{12.9}$ & $0.60_{0.04}^{0.12}$ & $7.22_{3.82}^{3.06}$ & $2.10_{0.46}^{1.12}$ & $1.32_{0.49}^{0.45}$ & 6.55 & $1.33(102)$ \\
\hline 9492 & $0.20_{0.10}^{0.09}$ & $0.15_{0.06}^{0.05}$ & $24.3_{18.3}^{94.8}$ & $0.58_{0.03}^{0.03}$ & $9.71_{2.80}^{2.42}$ & $1.90_{0.40}^{1.12}$ & $2.52_{0.93}^{0.78}$ & 7.09 & $0.96(68)$ \\
\hline 9816 & $0.12_{0.08}^{0.08}$ & $0.20^{\text {fr }}$ & $5.46_{3.74}^{7.08}$ & $0.61_{0.03}^{0.04}$ & $7.73_{1.16}^{2.24}$ & $1.49_{0.23}^{0.20}$ & $3.32_{0.72}^{0.62}$ & 7.70 & $1.00(74)$ \\
\hline 9493 & $0.16_{0.15}^{0.14}$ & $0.28_{0.08}^{0.07}$ & $15.0_{11.1}^{27.0}$ & $0.69_{0.12}^{0.31}$ & $3.37_{2.11}^{5.33}$ & $1.49_{0.26}^{0.47}$ & $3.20_{1.21}^{1.05}$ & 7.00 & $0.97(52)$ \\
\hline 9830 & $0.24_{0.17}^{0.15}$ & $0.23_{0.08}^{0.08}$ & $26.8_{20.2}^{88.8}$ & $0.62_{0.10}^{0.15}$ & $5.81_{4.10}^{5.05}$ & $1.94_{0.48}^{2.92}$ & $1.96_{0.99}^{1.02}$ & 6.19 & $0.76(47)$ \\
\hline 9831 & $0.19_{0.19}^{0.21}$ & $0.23_{0.23}^{0.13}$ & $27.0_{24.2}^{160}$ & $0.79_{0.17}^{0.25}$ & $4.57_{2.22}^{4.20}$ & $2.37_{0.80}^{4.65}$ & $2.80_{1.40}^{1.22}$ & 7.84 & $0.94(36)$ \\
\hline 9498 & $0.27_{0.08}^{0.09}$ & $0.23_{0.05}^{0.03}$ & $34.9_{15.9}^{32.3}$ & $0.71_{0.10}^{0.05}$ & $7.16_{1.85}^{4.28}$ & $2.63_{1.3}^{-}$ & $1.05_{0.74}^{0.87}$ & 7.14 & $1.14(78)$ \\
\hline 9859 & $0.22_{0.09}^{0.10}$ & $0.18_{0.04}^{0.06}$ & $42.3_{22.1}^{45.3}$ & $0.58_{0.03}^{0.03}$ & $10.8_{4.36}^{2.97}$ & $2.14_{0.76}^{-}$ & $1.32_{0.67}^{0.76}$ & 7.78 & 0.77 (76) \\
\hline 9482 & $0.31_{0.08}^{0.06}$ & $0.26_{0.03}^{0.03}$ & $31.3_{16.1}^{26.8}$ & $0.81_{0.11}^{0.13}$ & $2.66_{0.85}^{2.80}$ & $1.96_{0.31}^{0.35}$ & $1.63_{0.81}^{0.11}$ & 5.28 & 1.03 (106) \\
\hline Average & 0.22 & 0.22 & 25.6 & 0.67 & 6.56 & 2.00 & 2.12 & 6.95 & 0.99 \\
\hline
\end{tabular}

NOTE. - The "fr" indicates that the parameter was frozen at this value during spectral fitting. The $90 \%$ confidence level errors are quoted with a hyphen corresponding to an unconstrained error.

TABLE 5

PARAMETERS FOR THE MUTUAL WIND-WIND COLLISION BETWEEN SYSTEMS A AND B.

\begin{tabular}{lll}
\hline Parameter & System A & System B \\
\hline$M\left(\mathrm{M}_{\odot} \mathrm{yr}^{-1}\right)$ & $2.2 \times 10^{-6}$ & $5.8 \times 10^{-7}$ \\
$v_{\infty}\left(\mathrm{cm} \mathrm{s}^{-1}\right)$ & $2.14 \times 10^{8}$ & $2.30 \times 10^{8}$ \\
$\Xi$ & 0.06 & 0.35 \\
$\chi$ & 570 & 2860 \\
$f_{\mathrm{X}}\left(10^{-13} \mathrm{erg} \mathrm{s}^{-1} \mathrm{~cm}^{-2}\right)$ & 5.3 & 1.9 \\
$\eta$ & & 0.28 \\
$d_{\mathrm{sep}(\mathrm{A}+\mathrm{B})}\left(10^{12} \mathrm{~cm}\right)$ & \multicolumn{2}{c}{600} \\
$N_{\mathrm{H}}\left(\mathrm{cm}^{-2}\right)$ & \multicolumn{2}{c}{$1.3 \times 10^{20}$} \\
\hline
\end{tabular}

NoTE. - The values of $f_{\mathrm{X}}$ and $N_{\mathrm{H}}$ are calculated from Eqs. 1 and 8 respectively. The values of $\Xi$ for System A and System B were interpolated from the results of Pittard \& Stevens (2002) for the respective values of $\eta$. by mass transfer (for an alternative scenario see $\S 4.3$ ). Component $\mathrm{A} 1-\mathrm{O}$ is the faintest emitter (noting that component A1-O is bright for a brief period around periastron which is not sampled by our observations - see the $\phi_{\mathrm{A}}$ 's in Table 3, followed by the EWSs $\left(\approx 4.4 \times 10^{-13} \mathrm{erg} \mathrm{s}^{-1} \mathrm{~cm}^{-2}\right)$, both of which are fainter than the contribution from the MWC (Table 5). However, before making a detailed comparison with the best-fit values from the spectral fits we can improve our predictions by considering the energy dependence of the intrinsic and attenuated flux.

\subsection{Attenuated emission}

The range of preshock velocities evident in Fig. 4 w will cause the spectra from the different components to have different characteristic energies. We can examine the implications of this energy dependence by firstly calculating an intrinsic spectrum at the mean post-shock gas temperature using the MEKAL plasma code (Kaastra 1992; Mewe et al.|1995), where solar abundances are assumed Anders \& Grevesse 1989). Each intrinsic spectrum is then scaled so that the integrated $0.5-8 \mathrm{keV}$ fluxes are equal to the orbital phase dependent values, which for components $\mathrm{A} 1-\mathrm{O}$ and $\mathrm{B} 1-\mathrm{O}$ are shown in Fig. 4. The top panel of Fig. 5] shows the intrinsic spectra calculated for components A1-O, B1-O, the EWSs, and the MWC for Obs ID 6402 (see Table 3 for the respective orbital phases of systems A and B). The EWSs clearly contribute the softest spectrum. For the wind-wind collision shocks, the lower preshock velocity for component $\mathrm{B} 1-\mathrm{O}$ relative to the MWC and component A1-O also results in a slightly softer spectrum. Interestingly, although not the brightest emitter, the MWC has the hardest spectrum.

To estimate the impact of circumstellar absorption, a characteristic column density for the binary systems can be calculated using Eq(11) from Stevens et al. (1992),

$$
\bar{N}_{\mathrm{H}}=5 \times 10^{21} \frac{\dot{M}_{-7}}{v_{8}} \frac{\left(1+\eta^{1 / 2}\right)}{d_{12}}
$$

The expression for $\bar{N}_{\mathrm{H}}$ is for a binary system at quadrature where the winds are assumed to be at their terminal velocities ( $v_{\infty}$ 's are used to calculate $\eta$ in this case). When the stars are at quadrature all lines of sight to the emitting region will pass through the more powerful wind, which is assumed to be the dominant absorber. The orbital phase dependent characteristic column densities for systems A and B are $\simeq 4.9-9.9 \times 10^{21} \mathrm{~cm}^{-2}$ and $\simeq 4.5-5.6 \times 10^{21} \mathrm{~cm}^{-2}$, respectively. Note that in using Eq 8 we are essentially assuming the system is always at quadrature. To calculate the optical depth, we then multiply the total column density $\left(=\bar{N}_{\mathrm{H}}+N_{\mathrm{H} \text { ISM }}\right)$ by the opacity for gas at $10^{4} \mathrm{~K}$ calculated using version $c 08.00$ of Cloudy (Ferland 2000, see also Ferland et al. (1998)), where solar abundances are assumed (Anders \& Grevesse 1989). It is important to highlight that no circumstellar absorption is added for the EWSs as the $L_{\mathrm{X}} / L_{\mathrm{bol}}$ relation is for sources which have been corrected only for ISM absorption. Coincidently, the circumstellar absorption to the WCR between systems A and B is very small, so that this component essentially suffers only ISM absorption. The resulting attenuated spectra are displayed in the bottom panel of Fig.5. With the exception of the EWS emission, there is a consistent trend of a turnover energy of $\sim 1 \mathrm{keV}$. Furthermore, the biggest victims of energy dependent absorption are components A1-O and B1-O, which can be seen from a comparison of the intrinsic and attenuated spectra in Fig. 5

\subsection{Comparison to observations}

The model predictions and observations are in good agreement for a number of features. For instance, the X-ray emission from wind collision shocks can explain the hot plasma component derived from the spectral fits. Despite this, if all of the emitters in the system are active the total attenuated flux predicted by the model is $\sim 10-20$ times higher than the fluxes obtained from the spectral fits, with the dominant contribution to the model flux coming from the post-shock 

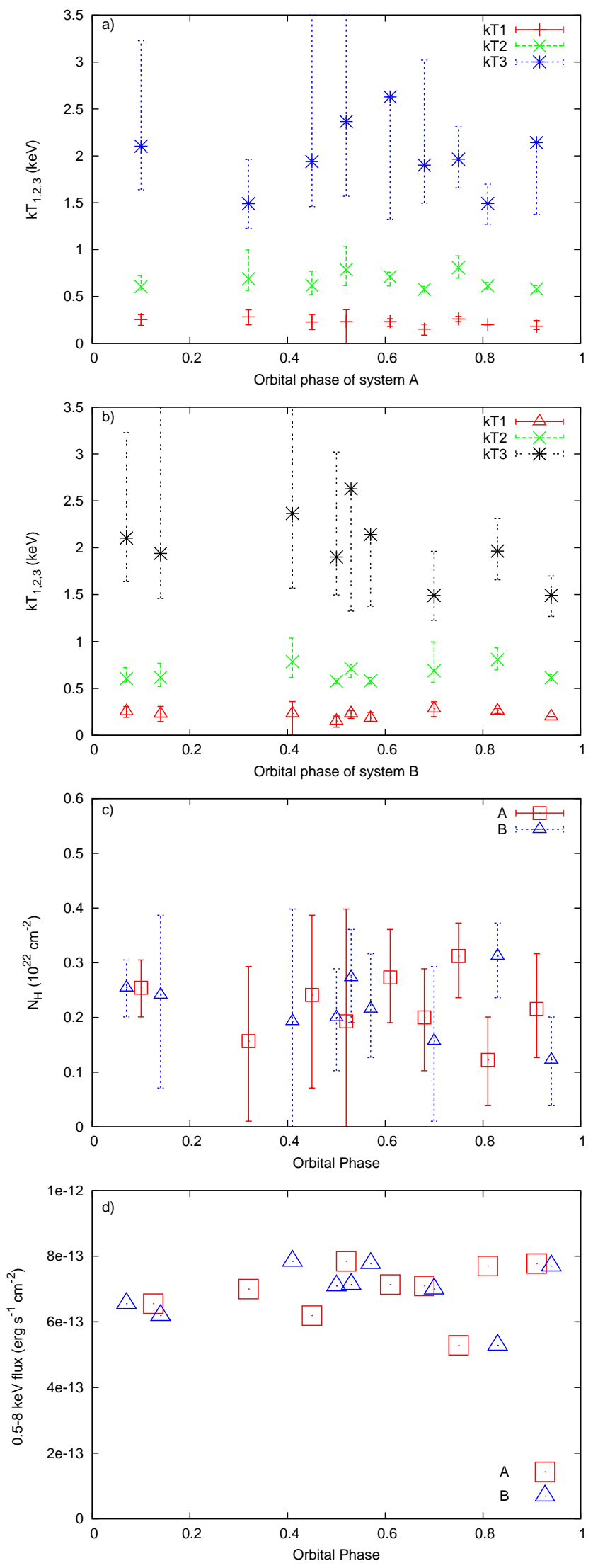

FIG. 3.- Optimal parameters attained from the spectral fits plotted against orbital phase for both system A and system B. From top to bottom: a) $k T$ 's for system A, b) $k T$ 's for system B, c) $N_{\mathrm{H}}$, and d) the $0.5-8 \mathrm{keV}$ flux. See also Table 4
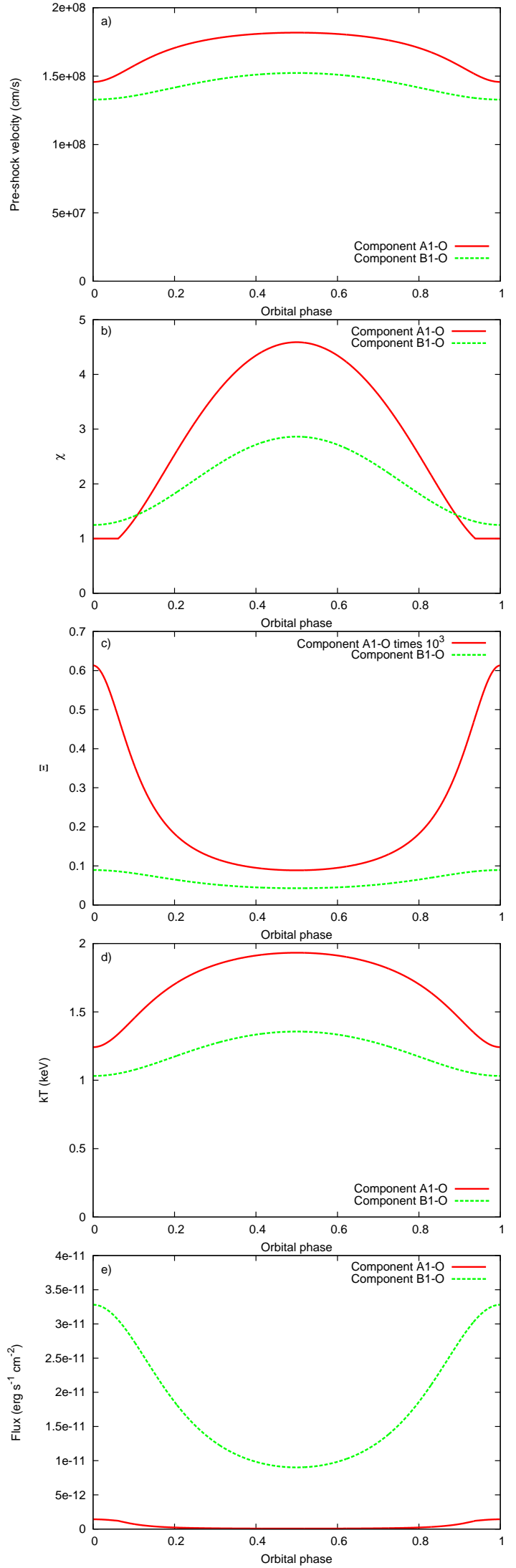

FIG. 4.- Variation with orbital phase of various parameters in the calculations of the X-ray luminosity generated in the multiple wind-wind collisions. From top left to bottom right: a) pre-shock velocity, b) cooling parameter $\chi$, c) fraction of wind kinetic power thermalized in the collision $\Xi$, d) the mean post-shock temperature $k T$, and e) the intrinsic X-ray flux due to wind-wind collisions from each component. $\chi$ 's are limited to 1 if the actual value is lower. The contributions from the post-shock gas of the different components are phased to their respective system, i.e. the contribution from component A1-O is phased to system A. Note that in the plot $\Xi_{\mathrm{A} 1}$ has been multiplied 

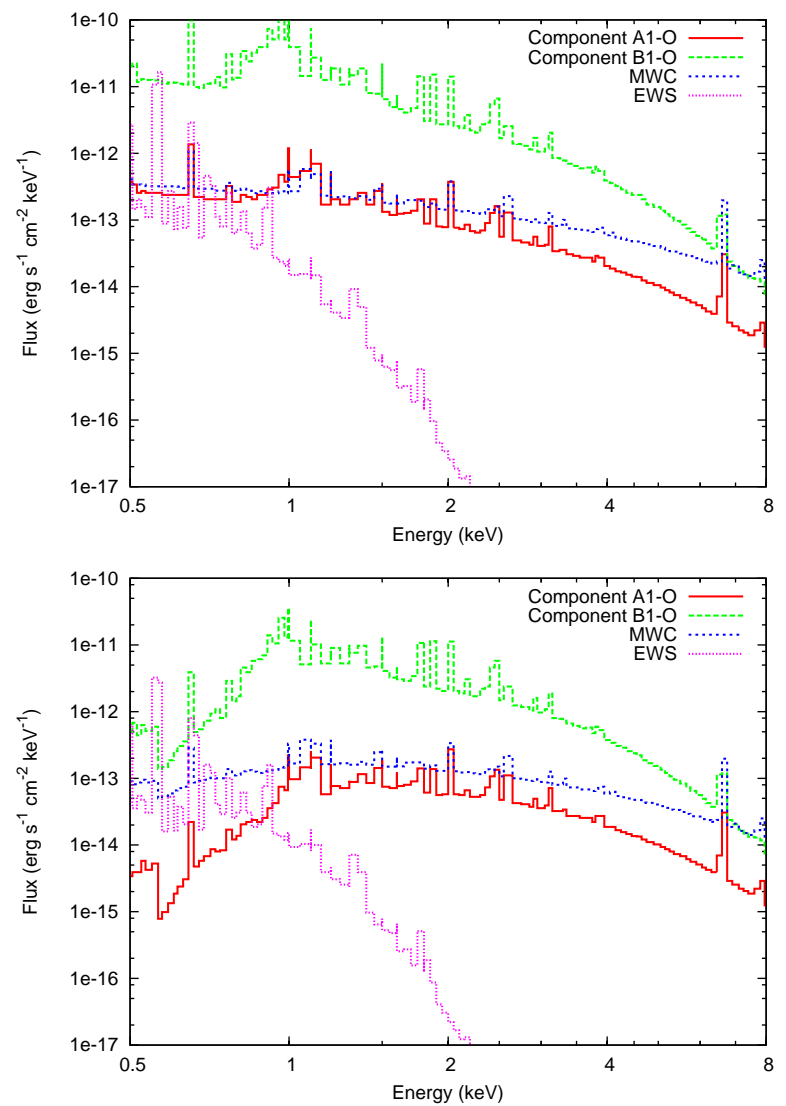

FIG. 5.- Intrinsic (top panel) and attenuated (bottom panel) synthetic 0.5$8 \mathrm{keV}$ X-ray spectra for Obs ID 6402.

gas of component B1-O (see bottom panel of Fig. 6). However, it is unclear whether the assumption of a normal wind for component B1-O is justified as Leung et al. (1979) suggested that this system is semi-detached. Numerical models of short period, massive star binary systems by Dessart et al. (2003) have shown that even a relatively small mass transfer rate $\left(\simeq 5 \times 10^{-6} \mathrm{M}_{\odot} \mathrm{yr}^{-1}\right)$ is unimpeded by the winds of the stars and can therefore disrupt the apex of the wind-wind collision region. To gain a better agreement between the model and observations it would seem necessary for some mechanism to kill-off the X-ray emission from system B; mass transfer is a viable option.

Recalling that a comparison of the spectra from QZ Car and those of single and binary stars in Carina showed that on the basis of the spectral shape and the derived plasma temperatures we cannot directly infer the presence of windwind collision shocks, one may be inclined to neglect the MWC emission also. Yet, if we only consider the X-ray emission from the individual stars (hereafter Scenario 1) there is a deficit between model and observation of a factor of $\sim 2-3$ (see Fig. 7]. Including the MWC emission (hereafter Scenario 2) remedies the shortfall, and a good agreement is attained when stellar mass-loss rates are reduced by a factor of 1.1. Noting that massive star winds are inhomo-

${ }^{9}$ Our adopted stellar parameters (Table 2] lead to an integrated $L_{\text {bol }}$ for QZ Car which is a factor of $\sim 2$ lower than determined by Povich et al. (2011). Although increasing $L_{\mathrm{bol}}$ by this factor will cause a corresponding increase in $L_{\mathrm{X}}$, the shortfall between Scenario 1 and the observed fluxes in Fig. 7 would not be remedied.
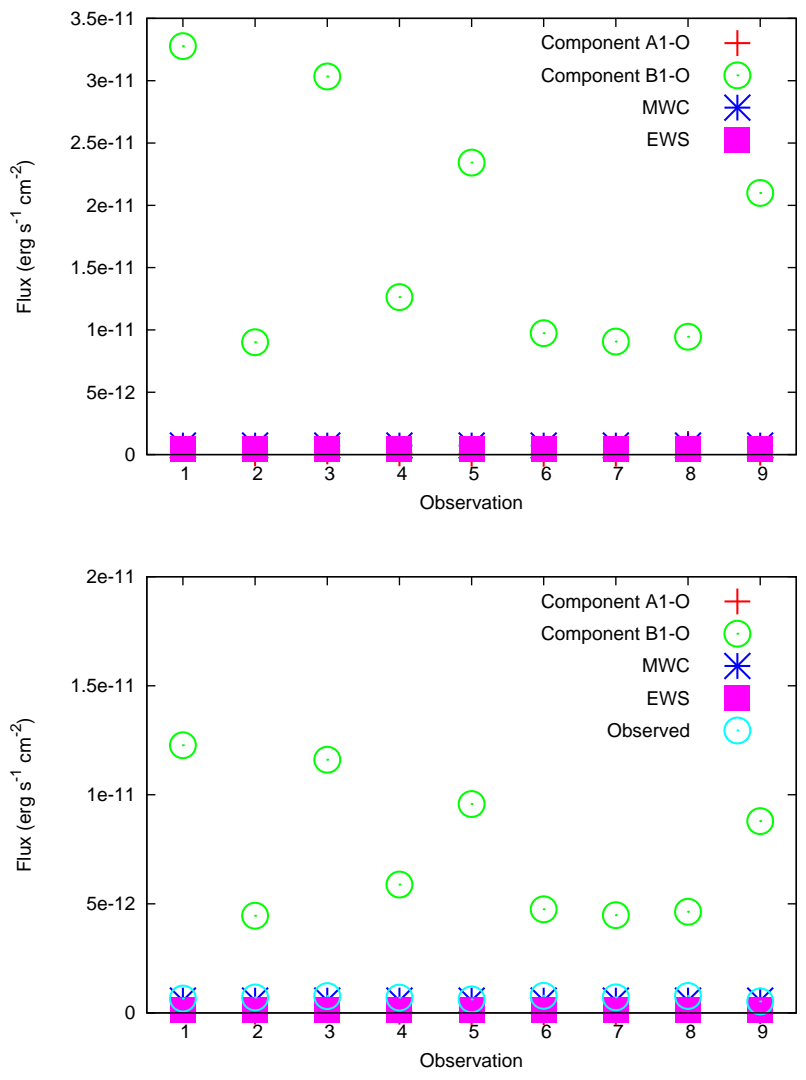

FIG. 6.- Variation of the intrinsic (top panel) and attenuated (bottom panel) $\mathrm{X}$-ray fluxes from wind-wind collisions. The fluxes are integrated from the synthetic spectra in the $0.5-8 \mathrm{keV}$ range. The observed fluxes are also plotted for comparison (see Table 4. Clearly, if one sums the contributions from all of the emitters in the model the flux exceeds the observed values derived from the spectral fits. Note the difference in scale between the plots.

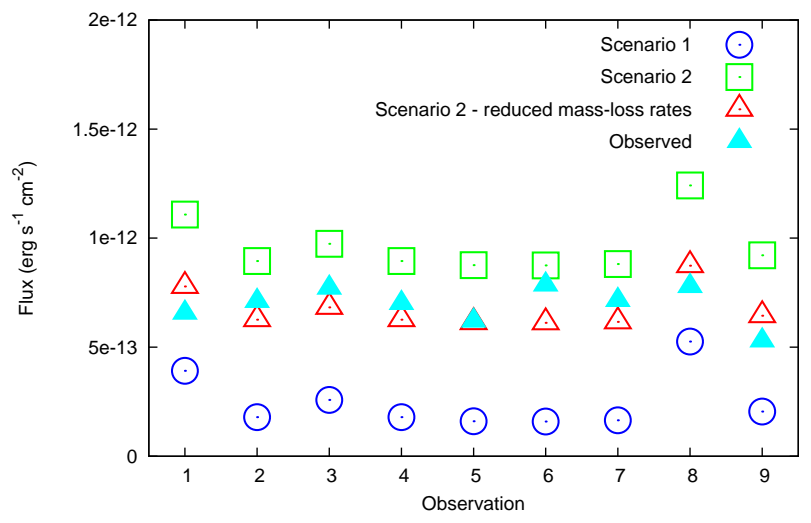

FIG. 7.- Integrated $0.5-8 \mathrm{keV}$ fluxes predicted by the model compared against the values attained from the spectral fits.

geneous (see Puls et al. 2008, for a recent review), and that results from detailed observational studies suggest that previous mass-loss rate estimates (e.g. Howarth \& Prinia 1989) require scaling down by factors of 2-5 (Bouret et al. 2003; Repolust et al. 2004; Markova et al. 2004; Fullerton et al. 2006; Moffat 2008; Waldron \& Cassinelli 2010), this seems to be a reasonably modest alteration. Notwithstanding the 
improved agreement between the model and observed fluxes, the average column derived from the spectral fits $(\sim 0.2 \times$ $10^{22} \mathrm{~cm}^{-2}$ ) is significantly higher than the value calculated for the MWC. This could be indicating two things. The first is that in using $\mathrm{Eq} 8$ we may be underestimating the column density to the shocked gas between systems A and B, or that a more detailed description of the X-ray emission and absorption to the individual stars is warranted.

Bearing in mind that the X-ray flux from the MWC $\propto$ $1 / d_{\text {sep } \mathrm{A}+\mathrm{B}}$ (since the shocked gas is adiabatic), we raise the question of whether a lower limit can be placed on $d_{\mathrm{sep} A+\mathrm{B}}$ if we make a slightly larger (but still reasonable) reduction in mass-loss rates? Proceeding with this approach the observed flux level can be approximately matched if we reduce $\dot{M}$ 's by a factor of 3 and decrease $d_{\mathrm{sepA}+\mathrm{B}}$ to $6 \times 10^{13} \mathrm{~cm}$, i.e. a factor of 10 smaller than the upper limit given by Nelan et al. (2004). This alternation in fact causes a negligible change to the column density calculated for the MWC. However, due to the uncertainty in the orbital eccentricity of system $\mathrm{AB}$ this is a somewhat tentative lower limit.

\section{DISCUSSION}

The presence of a hot plasma component with $k T \simeq 2 \mathrm{keV}$ in the spectral fits could be providing evidence for windwind collision shocks in QZ Car. However, to prevent our model predictions from considerably overestimating the observed flux we must suppress the prominent X-ray emission from system B. This is an interesting result as mass transfer in system B could provide an effective mechanism to disrupt the wind-wind collision region, and therefore our results support the previous suggestion by Leung et al. (1979) of mass transfer in system B (see also Morrison \& Conti 1980). Reassuringly, this result is unaffected by our adopted distance to QZ Car - we adopt a distance of $2.3 \mathrm{kpc}$ which differs from that of Southworth \& Clausen (2007) who quote a value of 2.8 $\mathrm{kpc}$. The net effect of using this slightly larger distance would be a reduction in the calculated fluxes by a factor of $\sim 0.67$, which would not affect our qualitative conclusions. We must note, however, that although additional emission from the MWC is required in our model, a more detailed description of the emission from the single star than adopted in this work may render this unnecessary. Furthermore, the ISM column density provides the dominant absorption to the MWC and the individual stars, therefore, a small increase/decrease in the ISM column could have implications for our model results.

This semi-analytical model has nevertheless provided a great deal of insight. Further progress will require detailed hydrodynamical modelling which should consider the following factors:

- The stellar separations in the binary systems are relatively small and therefore the interaction between the stellar radiation fields may affect the wind acceleration (e.g. inhibition or braking, Stevens \& Pollock 1994; Gayley et al. 1997) which would alter the resulting Xray flux (e.g. Parkin et al. 2009).

- Post-shock gas is in reality multi-temperature and more accurate comparisons against the observed spectra will require this to be taken into account.

- The nature of system B must be properly considered.

- We account for the radiative behaviour of the shocked gas through the $1 / \chi$ scaling in Eq. 1. However, the effect of radiative cooling on the dynamics and the X-ray emission is likely to be more complicated (e.g. Stevens et al. 1992; Mvasnikov et al. 1998; Antokhin et al. 2004; Pittard 2009; Parkin \& Pittard 2010; Parkin et al. 2011).

- Contrasting views exist as to the X-ray generation mechanism for single $\mathrm{O}$ type stars. For instance, recent high resolution analysis of $\mathrm{O}$ type stars has given evidence for a decrease in X-ray temperature in the stellar wind as one tends to larger radii (Waldron \& Cassinelli 2007). This poses questions for the classic picture of $\mathrm{X}$-ray generation by instability driven shocks, whereby higher X-ray temperatures are attained at larger radii at which point the flow has been accelerated somewhat (Owocki et al. 1988). Thus, it would be appropriate to assess these models in future work, in particular examining the spatial and energy dependence of the X-ray emission and absorption (e.g. Leutenegger et al. 2010).

\section{CONCLUSIONS}

We have presented a series of nine observations of the multiple star system QZ Car obtained with Chandra over a period of roughly 2 years. The spectral fits are characterised by cool, moderate, and hot temperature plasma components at $k T \simeq 0.2,0.7$, and $2 \mathrm{keV}$, respectively, a circumstellar absorption of $\simeq 0.2 \times 10^{22} \mathrm{~cm}^{-2}$, and an average flux of $\simeq 7 \times 10^{-13} \mathrm{erg} \mathrm{s}^{-1} \mathrm{~cm}^{-2}$. There appears to be no clear correlation between the fluxes and the orbits of the constituent binaries. The most compelling evidence for any correlation is between the high temperature thermal plasma component and the orbit of the O9.7 I + b2 v binary (system A), although due to limited statistics the high temperature plasma component is poorly constrained. Curiously, there is also a deficit between the X-ray flux expected from the single stars and that derived from the spectral fits.

A semi-analytical model of QZ Car was constructed. A stable momentum balance is not attained between the winds in either the O9.7 I + b2 v binary (system A) or the O8 III + $09 \mathrm{v}$ binary (system $\mathrm{B}$ ), and despite possessing the strongest stellar wind in QZ Car the O9.7 I star is a weak emitter (in terms of wind-wind collision emission) due to the relatively small fraction of its wind being shocked. The higher fraction of the primary star's wind being shocked in the O8 III $+09 v$ binary (system B) makes it the dominant emitter, although the magnitude of its X-ray emission exceeds the flux level derived from the spectral fits by more than a factor of 10 . The necessity of a disrupted wind-wind collision region in the O8 III + o9 $\mathrm{v}$ binary to bring the model results and observations into better agreement gives some compelling evidence in support of Leung et al. (1979)'s suggestion of mass transfer.

We conclude that the magnitude and lack of variability in the fluxes derived from the spectral fits can be well matched by a combination of X-ray emission from the individual stars and the mutual wind-wind collision between the two binary systems, albeit with stellar wind mass-loss rates reduced inline with the current consensus for inhomogeneous winds. The observed column density is, however, not well matched by the model. This may be indicating that a more complex prescription for the emission from the individual stars is required, or also that the column density calculation is not completely appropriate for the mutual wind-wind collision between the two binary systems. Our analysis places a somewhat tentative lower limit on the separation of the two binary 
systems of $\simeq 7 \mathrm{AU}$.

Future analysis would benefit from further observations. A follow-up X-ray observation with significant enough exposure time to allow a satisfactory fit with a three temperature plasma model with discrete absorption components could constrain the column density to the hot plasma. At radio wavelengths it may in fact be possible to resolve the separate emission peaks (e.g. Dougherty et al. 2005). However, the sensitivity of current instruments would require a long observation to attain sufficient statistics, which considering the timescale of the binary orbits may cause detailed structure to become smeared (Pittard 2010). With our results providing support for mass transfer in the O8 III + o9 v binary, multi-wavelength observations may reveal a far more complicated picture for QZ Car (e.g. $\beta$ Lyrae; Ignace et al. 2008).

We thank Rodolfo Barbá and the OWN Team (Barba et al. 2010) for providing new high-resolution optical spectroscopic results in advance of publication, Nolan Walborn for deriv- ing new spectral classifications from those data, as well as for useful discussions, and the referee for a helpful and insightful report. ERP was supported in part by a Henry Ellison Scholarship from the University of Leeds, and by a PRODEX XMM/Integral contract (Belspo). ERP also thanks Penn State University for their hospitality during a fruitful visit. JMP gratefully acknowledges funding from the Royal Society. AFJM is grateful for financial assistance from NSERC (Canada) and FQRNT (Quebec). YN acknowledges support from the Fonds National de la Recherche Scientifique (Belgium), the PRODEX XMM and Integral contracts, and the 'Action de Recherche Concertée' (CFWB-Académie Wallonie Europe). WLW acknowledges partial support from Chandra grant AR8-9003A. This work is supported by Chandra X-ray Observatory grant GO8-9131X (PI: L. Townsley) and by the ACIS Instrument Team contract SV4-74018 (PI: G. Garmire), issued by the Chandra X-ray Center, which is operated by the Smithsonian Astrophysical Observatory for and on behalf of NASA under contract NAS8-03060.

\section{REFERENCES}

Allen, D. A., \& Hillier, D. J. 1993, Proceedings of the Astronomical Society of Australia, 10, 338

Anders, E., \& Grevesse, N. 1989, Geochim. Cosmochim. Acta, 53, 197

Antokhin, I. I., Owocki, S. P., \& Brown, J. C. 2004, ApJ, 611, 434

Arnaud, K. A. 1996, in Astronomical Society of the Pacific Conference Series, Vol. 101, Astronomical Data Analysis Software and Systems V, ed. G. H. Jacoby \& J. Barnes, 17-+

Barbá, R. H., Gamen, R.,Arias, J. I., Morrell, N., Maíz Apellániz, J., Alfaro, E., Walborn, N. Sota, A., 2010, in Revista Mexicana de Astronomia y Astrofisica Conference Series, Vol. 38, Revista Mexicana de Astronomia y Astrofisica Conference Series, ed. Th. Rivinius \& M. Curé, 30-32

Bouret, J., Lanz, T., Hillier, D. J., Heap, S. R., Hubeny, I., Lennon, D. J., Smith, L. J., \& Evans, C. J. 2003, ApJ, 595, 1182

Broos, P., Townsley, L., Getman, K., \& Bauer, F. 2002, ACIS Extract, An ACIS Point Source Extraction Package, Pennsylvania State University, http://www.astro.psu.edu/xray/docs/TARA/ae _users _guide.html

Broos, P. S., Feigelson, E. D., Townsley, L. K., Getman, K. V., Wang, J., Garmire, G. P., Jiang, Z., \& Tsuboi, Y. 2007, ApJS, 169, 353

Broos, P. S., Townsley, L. K., Feigelson, E. D., Getman, K. V., Bauer, F. E., \& Garmire, G. P. 2010, ApJ, 714, 1582

Broos, P. S., et al. 2011, ApJS, submitted (CCCP Catalog Paper)

Chlebowski, T., \& Garmany, C. D. 1991, ApJ, 368, 241

Cox, A. N. 2000, Allen's astrophysical quantities, ed. Cox, A. N.

Davis, J. E. 2001, ApJ, 562, 575

De Becker, M., Rauw, G., Blomme, R., Pittard, J. M., Stevens, I. R., \& Runacres, M. C. 2005, A\&A, 437, 1029

Dessart, L., Langer, N., \& Petrovic, J. 2003, A\&A, 404, 991

Dougherty, S. M., Beasley, A. J., Claussen, M. J., Zauderer, B. A., \& Bolingbroke, N. J. 2005, ApJ, 623, 447

Ferland, G. J. 2000, in Revista Mexicana de Astronomia y Astrofisica Conference Series, Vol. 9, Revista Mexicana de Astronomia y Astrofisica Conference Series, ed. S. J. Arthur, N. S. Brickhouse, \& J. Franco, 153-157

Ferland, G. J., Korista, K. T., Verner, D. A., Ferguson, J. W., Kingdon, J. B., \& Verner, E. M. 1998, PASP, 110, 761

Fullerton, A. W., Massa, D. L., \& Prinja, R. K. 2006, ApJ, 637, 1025

Gayley, K. G., Owocki, S. P., \& Cranmer, S. R. 1997, ApJ, 475, 786

Herbst, W. 1976, ApJ, 208, 923

Howarth, I. D., \& Prinja, R. K. 1989, ApJS, 69, 527

Ignace, R., Oskinova, L. M., Waldron, W. L., Hoffman, J. L., \& Hamann, W. 2008, A\&A, 477, L37

Kaastra, J. S. 1992, Internal SRON-Leiden Report

Leung, K.-C., Moffat, A. F. J., \& Seggewiss, W. 1979, ApJ, 231, 742

Leutenegger, M. A., Cohen, D. H., Zsargó, J., Martell, E. M., MacArthur,

J. P., Owocki, S. P., Gagné, M., \& Hillier, D. J. 2010, arXiv:1007.0783

Markova, N., Puls, J., Repolust, T., \& Markov, H. 2004, A\&A, 413, 693

Martins, F., Schaerer, D., \& Hillier, D. J. 2005, A\&A, 436, 1049

Mason, B. D., Gies, D. R., Hartkopf, W. I., Bagnuolo, Jr., W. G., ten Brummelaar, T., \& McAlister, H. A. 1998, AJ, 115, 821
Mayer, P., Niarchos, P. G., Lorenz, R., Wolf, M., \& Christie, G. 1998, A\&AS, 130, 311

Mayer, P., Lorenz, R., Drechsel, H., \& Abseim, A. 2001, A\&A, 366, 558

Mewe, R., Kaastra, J. S., \& Liedahl, D. A. 1995, Legacy, 6, 16

Moffat, A. F. J. 2008, in Clumping in Hot-Star Winds, ed. W.-R. Hamann, A. Feldmeier, \& L. M. Oskinova, 17-+

Morrison, N. D., \& Conti, P. S. 1979, in IAU Symposium, Vol. 83, Mass

Loss and Evolution of O-Type Stars, ed. P. S. Conti \& C. W. H. de Loore, 277-280

Morrison, N. D., \& Conti, P. S. 1980, ApJ, 239, 212

Myasnikov, A. V., Zhekov, S. A., \& Belov, N. A. 1998, MNRAS, 298, 1021

Nazé, Y. 2009, A\&A, 506, 1055

Nazé, Y., et al. 2011, ApJS, submitted (CCCP Massive Star Lx/Lbol Paper)

Nelan, E. P., Walborn, N. R., Wallace, D. J., Moffat, A. F. J., Makidon,

R. B., Gies, D. R., \& Panagia, N. 2004, AJ, 128, 323

Oskinova, L. M. 2005, MNRAS, 361, 679

Owocki, S. P., Castor, J. I., \& Rybicki, G. B. 1988, ApJ, 335, 914

Owocki, S. P., \& Cohen, D. H. 1999, ApJ, 520, 833

Parkin, E. R., \& Pittard, J. M. 2008, MNRAS, 388, 1047

-. 2010, MNRAS, 406, 2373

Parkin, E. R., Pittard, J. M., Corcoran, M. F., Hamaguchi, K., \& Stevens, I. R. 2009, MNRAS, 394, 1758

Parkin, E. R., Pittard, J. M., Corcoran, M. F., \& Hamaguchi, K. 2011, ApJ, 726, 105

Pittard, J. M. 2009, MNRAS, 396, 1743

—. 2010, MNRAS, 403, 1633

Pittard, J. M., \& Parkin, E. R. 2010, MNRAS, 403, 1657

Pittard, J. M., \& Stevens, I. R. 1997, MNRAS, 292, 298

-. 2002, A\&A, 388, L20

Pollock, A. M. T. 1987, ApJ, 320, 283

Povich, M. S., et al. 2011, ApJS, submitted (CCCP Massive Star Candidates Paper)

Prinja, R. K. 1989, MNRAS, 241, 721

Puls, J., Vink, J. S., \& Najarro, F. 2008, A\&A Rev., 16, 209

Repolust, T., Puls, J., \& Herrero, A. 2004, A\&A, 415, 349

Smith, N. 2002, MNRAS, 337, 1252

-. 2006, MNRAS, 367, 763

Smith, R. K., Brickhouse, N. S., Liedahl, D. A., \& Raymond, J. C. 2001, ApJ, 556, L91

Southworth, J., \& Clausen, J. V. 2007, A\&A, 461, 1077

Stevens, I. R., Blondin, J. M., \& Pollock, A. M. T. 1992, ApJ, 386, 265

Stevens, I. R., \& Pollock, A. M. T. 1994, MNRAS, 269, 226

Townsley, L. K., Broos, P. S., Feigelson, E. D., Garmire, G. P., \& Getman,

K. V. 2006, AJ, 131, 2164

Townsley, L. K., et al. 2011, ApJS, submitted (CCCP Intro Paper)

Vink, J. S., de Koter, A., \& Lamers, H. J. G. L. M. 2000, A\&A, 362, 295

Walborn, N. R. 1995, in Revista Mexicana de Astronomia y Astrofisica

Conference Series, Vol. 2, The Eta Carinae Region: A laboratory of

Stellar Evolution, ed. V. Niemela, N. Morrell, \& A. Feinstein, 51-+

Waldron, W. L., \& Cassinelli, J. P. 2007, ApJ, 668, 456 
